\title{
La détermination du moment d'inertie de groupe hydro-électrique
}

\author{
M. CUENOD (1)
}

INGÉNIEUR E.P.F. ZURICH

\section{The determination of the moment of inertia of a hydro-power unit}

English synopsis, p. 106.

\section{1. - INTRODUCTION}

L'équipement des centrales hydro-électriques nécessite une collaboration judicieuse entre tous ceux qui y participent. Une des questions qui, en particulier, doit faire l'objet d'une entente entre électricien et mécanicien, concerne le choix du moment d'inertie à domner aux masses tournantes de l'installation considérée.

Considérons que la puissance active et réactive, la vitesse nominale, la vitesse d'emballement, la tension et la fréquence d'un groupe hydro-électrique dont on fait le projet, soient données. Le «PD naturel » est celui que le constructeur du générateur serait amené à choisir de façon à obtenir les conditions économiques et techniques les plus avantageuses tant du point de vue électrique que mécanique.

En première approximation, ce $\mathrm{PD}^{2}$ naturel se laisse déterminer par la formule suivante :

$$
\mathrm{PD}^{2}{ }_{\text {nat }}=\mathrm{K} 10^{\mathrm{i}} \frac{\mathrm{N}}{\mathrm{n}^{2}}
$$

avec :

$$
\begin{aligned}
\mathrm{K} & =1,5-3 . \\
n & =\text { vitesse en } \mathrm{t} / \mathrm{mn} . \\
\mathrm{N} & =\text { puissance électrique } \mathrm{kVA} . \\
\mathrm{PD}^{2} & =\text { en } \mathrm{kgm}^{2} .
\end{aligned}
$$

La grandeur du $\mathrm{PD}^{2}{ }_{\text {nat }}$ ne doit pas etre considérée comme une valeur absolue; elle peut varier dans une assez grande marge selon le type de la construction choisie et selon les constructeurs.

(1) Ingénieur à la Société Ofinco, 2, rue de la Tertasse, Genève (Suisse).
D'autres facteurs relevant des conditions de marche du groupe doivent être également pris en considération pour la détermination du PD² d'un groupe hydro-électrique, en particulier la survitesse à la suite d'une décharge brusque du groupe, la stabilité du réglage de vitesse, la tenue de la fréquence et la répartition de la charge à la suite d'une décharge partielle. Dans beaucoup de cas, le PD $^{2}$ naturel suffit pour satisfaire aux exigences qu'imposent ces conditions de marche. Cependant, dans d'autres cas, on peut être conduit à choisir un $\mathrm{PD}^{2}$ qui soit un mul-tiple du $\mathrm{PD}^{2}$ naturel, soit en augmentant le diamètre du rotor ou en le rendant plus massif, soit en lui accouplant un volant. La première de ces mesures entre surtout en ligne de compte pour les groupes tournant relativement lentement, tandis que l'adjonction d'un volant doit être envisagée pour des groupes tournant relativement rapidement. La sollicitation mécanique due à la force centrifuge limite dans ce deuxième cas le diamètre que l'on peut domer au rotor et l'exiguité de la place à disposition ne permet pas de l'alourdir à volonté.

L'augmentation du PD² ne constitue pas en soi une difficulté d'ordre constructif; son inconvénient est d'ordre économique : elle augmente le prix et les pertes de la machine en question et cela surtout pour les groupes ayant une vitesse relativement élevée, car d'une façon générale, pour une machine électrique, plús le rapport entre la longueur du fer et l'alésage du stator est faible, plus cette machine est économique.

L'augmentation du prix est particulièrement rapide à partir d'une certaine valeur du $\mathrm{PD}^{2}$ correspondant au diamètre maximal admissible 
du rotor par suite de considérations mécaniques. Par le fait des pertes par ventilation, le rendement de la machine diminue Iorsque le $\mathrm{PD}^{2}$ augmente, et cela en particulier pour une marche à charge réduite.

II ressort de ce rapide exposé des différents fucteurs dont il faut tenir compte pour le choix du PD' d'un groupe hydro-électrique, que ce choix est une question très complexe qui doit etre envisagée sous tous ces aspects, aussi bien economiques que techniques. Car, si une augmentation du $\mathrm{PD}^{2}$ permet de diminuer le prix du côté hydraulique, elle augmente celui du générateur et s'accompagne d'un abaissement de son rendement.

De nombreux travaux, tant de la part des constructeurs de générateurs que de la part des lurbiniers, ont été déjà consacrés à l'examen de celte question (1).

Si nous souhaitons a notre tour apporter une certaine contribution à cette étude, c'est que les considérations suivantes nous y ont engagés :

1. Certaines nouvelles installations hydroélectriques sont prévues actuellement qui présen-

1) Voir en particulier :

-.. Contribution à l'étude des régulateurs de vitesse. Considéation sur le problème de la stabilité.

J. Gaden, Ed. La Concorde, Lausanne, 1945.

- Influence de certaines caractéristiques intervenant dans la condition de stabilité.

D. Gaden et P. NeEser, La Houllle Blanche, maijuin 1948 .

- Influence de l'inertie de l'eau sur la stabilité d'un groupe hydro-électrique.

P. Alueras, La Houlle Blanche, nov. 1945, janv. 1946 , mars 1946.

- Drehzahlregelung ron Wasserturbinen.

Th. STET, Schw, Bauzeitung, sept-octoble 1947.

- Du choix de quelques caractéristiques des alternateurs. J. Ricalexs, Bulletin de la Société Française des Electriciens, juillet 1947.

-.-. Délermination du PD2 de groupe hydro-électrique. J. ChExals, Bulletin de la Societe Frangaise des Electriciens, novembre 1947.

- Remarque sur les relations entre survitesse, surpression et stabilité des groupes hydro-électriques.

P. Almenas, Bulletin de la Societé Francaise des Electriciens.

-. Sur les alternatems de grande puissance pour centrales hydrauliques.

E. Guntrard, Revue Brown Boveri, juillet-août 1949 ,

- Influence des phénomènes de coups de béliers sur le réglage de la vitesse des turbines hydrauliques.

M. Cúxon, La Houille Blanche, mars-avril 1949.

- Du choix du moment d'inertie des groupes hydroélectriques.

D. Gaden et J. Ricalens, Rapport n 102, Gigre, 1948.

- L'influence de la vitesse d'emballement et du moment de giration sur le poids et le cout des alternateurs triphasés entrainés par turbine hydraulique à grande vitesse spécifique et la question de l'essai d'emballement des roues polaires.

H. Schuten, Rapport no 116, Cigre, 1948. tent des conditions hydrauliques particulièrement défavorables, c'est-à-dire avec un temps caractéristique $\theta$ élevé :

Il est connu en effet que, en première approximation, ces conditions hydrauliques peuvent être appréciées à l'aide du temps caractéristique de la conduite 0 , tel que :

$$
\theta=\frac{g \mathrm{H}}{\mathrm{I} \cdot v_{m}}
$$

$\mathrm{L}=\mathrm{Longueur}$ de la conduite (en $\mathrm{m}$ ).

$y_{m}=$ Vitesse moyenne de l'eau dans la conduite à pleine charge $(\mathrm{en} \mathrm{m} / \mathrm{s}$ ).

$\mathrm{H}=$ Hauteur de chute (en $\mathrm{m}$ ).

$g=$ Constante d'accélération $-9,81 \mathrm{~m} / \mathrm{s}^{2}$.

Ainsi, plus la longueur de la conduite et plus la vitesse de l'eau dans la conduite (proportionnelle au débit) sont élevées, et plus la hauteur de chute est faible, plus les conditions hydrauliques sont défavorables.

Il en résulte que, selon les normes actuelles, on soit conduit à augmenter considérablement le $\mathrm{PD}^{2}$ de groupes hydro-électriques en question au-delà de Feur PD² naturel, ce qui constitue au point de vue économique une servitude qu'il faut s'efforeer de limiter le plus possible.

2. - Par suite du développement de linterconnexion, la marche sur un réseau séparé apparait de plus en plus comme un cas exceptionnel; nous verrons que, dans le cas de la marche en parallèle, la stabilité ne dépend pas en premier lieu du choix du $\mathrm{PD}^{2}$ des groupes interconnectés, et que, d'autre part, pour le cas qui tend à devenir de plus en plus rare de marche sur réseau séparé, avec charge indépendante de la fréquence, ou même diminuant avec la fréquence, et réglage à tension constante, la stabilité peut être assurée autrement que par une augmentation du moment d'inertie du groupe, en réglant la charge électrique de facon appropriée.

3. - Certaines méthodes modernes de calcul pour l'ćtude des réglages automatiques permettent d'obtenir une meilleure approximation des conditions de stabilité en tenant compte de certains facteurs que les méthodes classiques devaient négliger pour ne pas rendre les calculs inextricables. Il est ainsi possible de réduire le facteur de sécurité qui devait englober l'ensemble de ces facteurs et de serrer le problème de plus près.

Nous mous proposons de passer en revue les différents facteurs qui interviennent pour le choix du PD de groupe hydro-électrique et de faire apparaitre certains de ces facteurs sous un jour un peu nouveau. 


\section{DISCUSSION DES FACTEURS QUI PEUVENT CONDUIRE A UNE AUGMENTATION DU PD' ${ }^{2}$ AU-DELA DU PD' NATUREL}

\section{A. Survitesse à la suite d'une décharge brusque.}

Lorsqu'à la suite du jeu de la protection le disjoncteur qui accouple le générateur à sa charge est brusquement ouvert, le couple électrique s'annule pratiquement instantanément; par suite de l'inertie de l'eau et du danger des coups de bélier, il ne peut en être de mème du couple moteur; en première approximation on peut admettre que la fermeture du vannage s'effectue linéairement par rapport au temps, ce qui, seion M. A. Rrbaux (2), conduit à la formule suivante pour la détermination du PD².

$$
\mathrm{PD}^{2}=\left(\frac{606}{n}\right)^{2} \frac{\mathrm{TN} \cos \varphi}{2 e+e^{2}}
$$

avec :

$\mathrm{N}=$ puissance unitaire (kVA).

$n=\operatorname{vitesse}(\mathrm{t} / \mathrm{mn})$.

$e=\frac{\Delta n}{n}=$ survitesse relative maximale.

$\mathrm{T}=$ temps de fermeture du vannage $(\mathrm{s})$.

$P D=$ en $\mathrm{kg} \mathrm{m}^{2}$.

La limite inférieure du temps de fermeture $\mathrm{T}$ est imposée par le danger des coups de bélier. Il est connu que la surpression à laquelle est soumise une conduite forcée à la suite d'une fermeture linéaire de son vannage est caractérisée par le quotient suivant :

$\frac{\text { temps caractéristique de la conduite }}{\text { temps de fermeture du vannage }}=\frac{\theta}{\mathrm{T}}=\frac{\mathrm{L} v_{m}}{g \mathrm{H} \mathrm{T}}$

Nous voyons donc que, en ce qui concerne le danger de survitesse, le choix du PD² est lié aux facteurs suivants : vitesse et survitesse admissibles, puissance unitaire, temps de fermeture du servo-moteur ' $T$, lui-même imposé par les limites qu'il faut imposer au coup de bélier, phénomène qui dépend de la disposition de la conduite forcée (hauteur de chute $\mathbf{H}$, longueur $\mathbf{L}$, diametre), et auquel on peut obvier par certains dispositifs de la turbine : orifice compensateur, déchargeur et déflecteur.

\section{B. Stabilité du réglage de vitesse}

La figure 1 représente le principe du réglage d'un groupe hydro-électrique. Le générateur $G$ est entrainé par la turbine $\mathrm{T}$, alimentée par une conduite de longueur $L$ et de hauteur $H$. Sa vilesse est mesurée par la course du régleur $R$

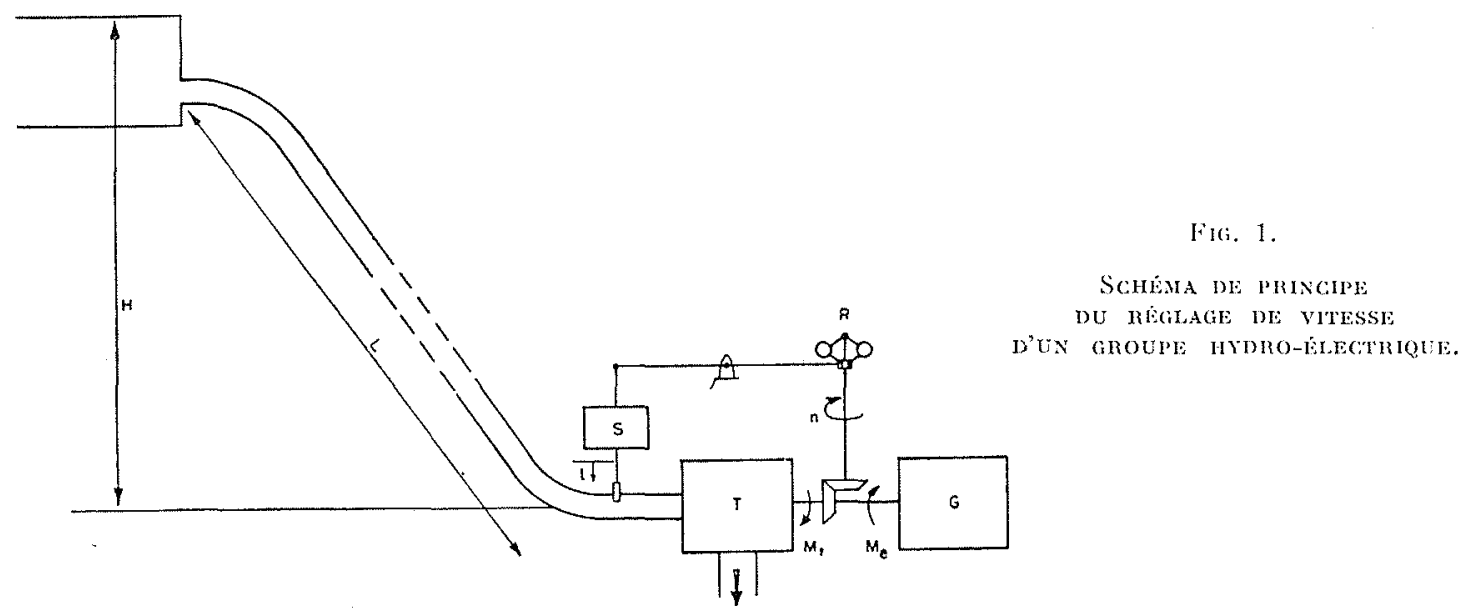

qui commande, par l'intermédiaire du servo-moteur $S$, l'ouverture $l$ de la turbine.

(2) Rubaux. - Régulateur de vitesse. Edition «La Moraine $\gg$, Geneve, 1947, p. 8 .
La stabilité du réglage de vitesse dépend des facteurs suivants :

Caractéristique du dispositif de réglage (régleur + servo-moteur) compte tenu des dispositifs 
de stabilisation (accélérometre ou statisme passager).

Caractiristique du système hydraulique (débit, longueur de la conduite, hauteur de chute, chisticite de la conduite et de l'eau).

\section{Giracteristique de la turbine.}

Caractéristique du générateur, compte tenu du genre de la charge et de son excitation (générateur accouplé à un réseau, directement ou par l'intermédiaire d'une longue ligne, marche indépendante avec charge ohmique ou induclive, riglage à tension constante ou à tension dépendante de la fréquence).

Le nombre et la complexité de ces facteurs rendent l'étude de la stabilité avec la méthode classique de Hurwrtz assez malaisée dès que l'on ne se contente plus d'une première approximation. Les difficultés du calcul sont beaucoup réduites, si l'on utilise certains procédés modernes de calcul faisant usage des transformations de LAPlace et du critère de Nyouist.

Les principes théoriques, sur lesquels reposent ces méthodes modernes de calcul, ont fait l'objet de plusieurs publications (3). Nous voulons rappeler seulement que les caractéristiques dynamiques d'un des éléments du circuit de réglage peuvent être définies par une équation différentielle, par sa courbe de réponse ou par sa courbe de Nyourst.

La courbe de réponse est donnée par la variation de grandeur de sortie dudit élément à la suite d'une variation impulsive de la grandeur d'entrée.

La courbe de NyQurst donne la phase et l'anplitude des variations de la grandeur de sortie
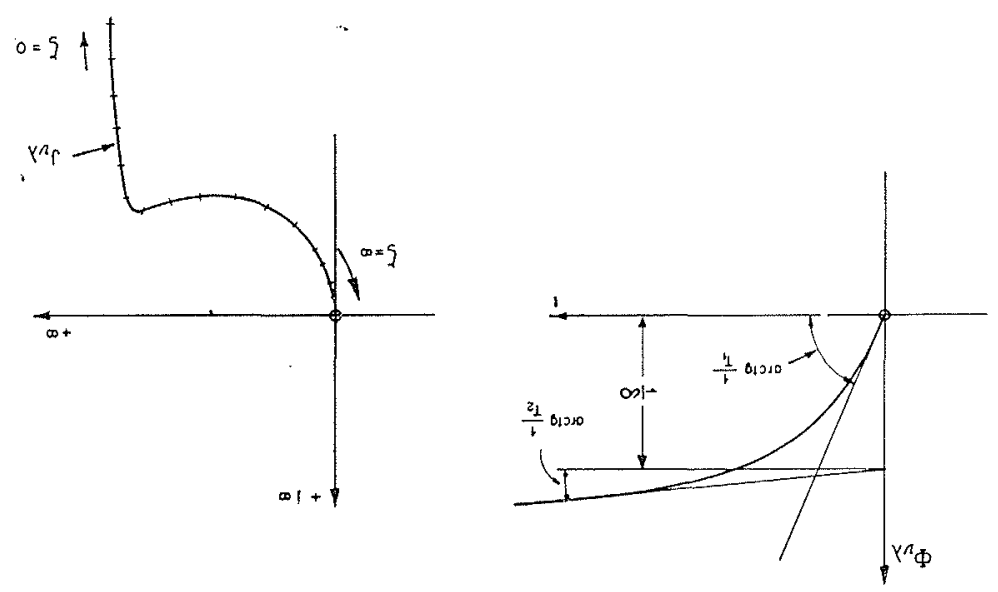

FIG. 2.

Caractínistoule DYNamiQue DU DISPOSITIF DE REGLAGE. résultant d'une oscillation harmonique de la grandeur d'entrée.

A l'aide des transformations de Laplace, il est aisé de déterminer la courbe de Nyouist d'un élément de circuit de réglage dont on connait la courbe de réponse on l'équation différentielle.

Le critere de Nroust énonce que si la courbe de Nyoust de chaque elément du circuit de réglage est connue, et que lon a déterminé la courbe de NYQUisT résultante du circuit de réglage en faisant le produit des courbes de Nyouist partielles entre elles, on peut déduire la stabilité du réglage, en considérant la position de cette courbe de Nyouist dessince dans le plan complexe par rapport au point :

$$
(+1, j O) \text { (avec } j=\sqrt{-1}) \text {. }
$$

Nous allons déterminer les caractéristiques dynamiques des difrérents organes du circuit de réglage de la vitesse d'un groupe hydro-électrique.

\section{Dispositif de réglage}

Par suite de la stabilisation (accéléromètre ou statisme passager) à la suite d'une variation brusque de la vitesse, le dispositif de réglage astatique effectue tout d'abord une correction relativement rapide, proportionnelle à l'écart de vitesse, puis continue sa course plus lentement

(3) Voir en particulier:

W. Frey : «Sur une généralisation des critères de stabilité de Nyquist et de Leonhard $\gg$.

Revue Brown Boveri, mars 1946.

C. Nasse : "Le circuit de régulation».

Actualité Scientifique et Industrielle, Paris, 1949.

C. Gammahe : «Servomécanisme et régulateur». Lev. gén. d'électr., janv. 1949.

M. Cuśxon: « Etude des propriétés d'un réglage automatique $\gg$.

Bulletin techn. de la Suisse Romande, 26 avril, 10 mai 1947 .

M. Demontrignen et P. Lefève : « Une nouvelle méthode harmonique d'étude de la stabilité des systèmes linéaires 》.

Revue Gén. d'Electricité, juillet 1949. 
de façon linéaire, tant que subsiste l'écart de vitesse; il en résulte la courbe de réponse représentée par la figure $2 a$, que nous supposons avoir été relevée expérimentalement et dont nous déterminons en première approximation l'expression mathématique :

$$
\Phi_{n \lambda}=\frac{1}{\delta}\left(1-e^{-\frac{t}{T_{1}}}\right)+\frac{t}{T_{2}}
$$

$y=$ variation relative de la vitesse.

$\lambda=$ variation relative de la course du vannage.

$\grave{\partial}=$ statisme passager résultant.

$\mathrm{T}_{1}=$ temps de réaction - - temps que met le servo-moteur pour parcourir $a \%$ de sa course après un écart de vitesse de $a \%$ pendant la premiere partio de son action.

$\mathrm{T}_{2}=$ temps d'amortissement - temps que met le servomoteur pour parcourir $a \%$ de sa course après un icartement de vitesse de a \%, la vitesse du díplacement etant constante.

La fonction de transfert s'obtient en appliquant la transformation de Laplace à l'équation de la courbe de réponse:

$$
\rho_{p \lambda}=\frac{1}{\delta} \frac{1}{p \mathrm{~T}_{1}+1}+\frac{1}{p \mathrm{~T}_{2}}
$$

L'expression de la courbe de Nrovis't s'obtient en remplaçant l'opérateur $p$ par $i t$ dans l'expression de la fonction de transfert.

$$
\begin{aligned}
j & =\sqrt{-1} . \\
\zeta & =\text { pulsation des oscillations. } \\
J_{\nu, \lambda} & =\frac{1}{\delta} \frac{1}{j \zeta \mathrm{T}_{1}+1}+\frac{1}{j \zeta \mathrm{T}_{2}}
\end{aligned}
$$

elle est représentée par la courbe de la figure $2 b$ sous forme vectorielle et par les courbes $J_{\nu \lambda}$ et $\psi_{\nu \lambda}$ des figures 6 et 7 sous forme cartésienne.

\section{GROUPE TURBINE-GENERATEUR}

L'équation différentielle des masses tournantes du groupe est classique :

$$
\mathrm{T}_{a} \frac{d \nu}{d t}+\mu_{e}=u_{t}
$$

$\mu_{e}=$ variation relative du couple résistant électrique.

$\mu_{i}=$ variation relative du couple-moteur exercé par la turbine.

$\mathrm{T}_{a}=$ constante d'accélération $=\left(\frac{n}{606}\right)^{2} \frac{\mathrm{PD}^{2}}{\mathrm{~N} \cos ?}$

( $n$ en $\operatorname{trs} / \mathrm{mm}, \mathrm{N}$ en liva, $\mathrm{PD}^{2}$ en $\mathrm{kg} \mathrm{m}^{2}$ ).

A titre de référence, nous déterminons la constante d'accélération qui correspond au $\mathrm{PD}^{2}$ naturel :

$$
\mathrm{T}_{a b}=\left(\frac{n}{606}\right)^{2} \cdot \frac{\mathrm{K} 10^{6} \mathrm{~N}}{\mathrm{~N} \cos \varphi n^{2}}=2,84 \frac{\mathrm{K}}{\cos \varphi}
$$

En considérant que $K$ est compris entre 1,5 et 3 , et $\cos$ o entre 0,8 et 0,9 , nous voyons que la constante d'accólération se tient pratiquement toujours entre 5 et 10 secondes.

\section{Détermination du couple résistant $\omega_{0}$.}

La valeur du couple résistant $\mu_{0}$ dépend essentiellement du genre de la charge et de l'exeitation.

Si le générateur est en marche indépendante, la relation entre le couple résistant et la vitesse en régime permanent est donnée par les caractéristiques du générateur représentées par la famille de courbes : $G_{1}, G_{2}, G_{3} \ldots G_{n}$ de la figure 3

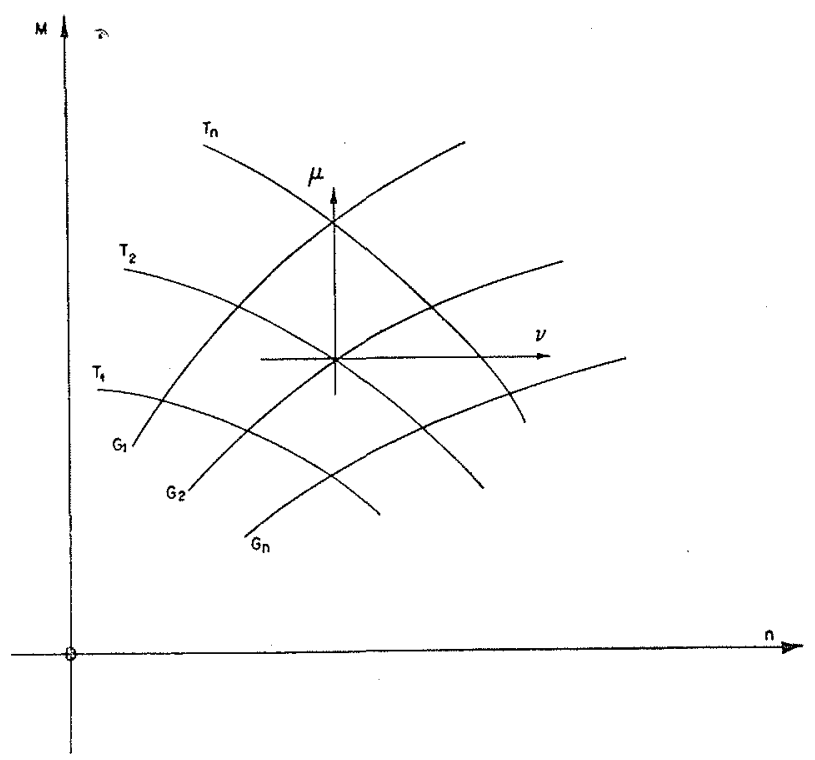

FIG. 3.

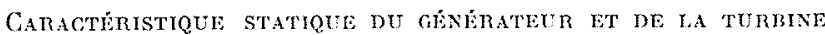


Nous obtenons :

$$
\mu_{4}=\varphi_{\nu \mu_{e}} \cdot \nu
$$

Avee:

$\|_{z \mu_{b}}=$ fonction du transfert du couple résistant en fonction de la vitesse.

En premiere approximation, en négligeant les phénomènes transitoires du réglage de la tension, on peut remplacer la caractéristique du générateur par sa tangente au point de régime. II en résulte:

$$
v_{\nu \mu_{c}}=\frac{\mu_{c}}{\nu}=\operatorname{tg} \alpha_{c}
$$

avec: :

Ig $x_{c}=-1$ réglage à puissance constante.

$\operatorname{tg} x_{c}=0$ réglage à pulissance augmentant proportionnellement à la fréquence.

$\lg \alpha_{0}=1$ réglage à puissance augmentant proportionnellement au carré de la fréquence.

Si le générateur est accouplé à un réseatu infiniment puissant, il est connu que le couple synchronisant est, en première approximation, proportionnel au déphasage interne du générateur, e'est-à-dire à l'intégrale de l'écart de vitesse, plus un facteur d'amortissement (4) :

$$
w_{u}={ }^{r} \mathrm{~T}_{u}\left(\Omega^{2} \int y a t+\frac{2 y}{\mathrm{~T}_{u}}\right)
$$

avec :

$Q^{2}=$ pulsation propre du générateur.

$\mathrm{T}_{y}=$ constante d'amortissement du genérateur.

d'où :

$$
э_{\nu \mu_{c}}=\frac{\mathrm{T}_{a} \Omega *}{p}+\frac{2 \mathrm{~T}_{a}}{\mathrm{~T}_{\prime \prime}} .
$$

\section{Détermination du couple motent ${ }_{L_{1}}$.}

Le couple moteur est dépendant du débit, de la pression de l'eau, de la vitesse, de la caracléristique et du rendement de la turbine. Débit et pression dépendent des variations de l'ouverture. La relation entre la vitesse et le couple moteur est donnée par la caractéristique cou-

(4) Voir M. Cuénon: « Etudes des propriécés d'un réglage automatique $»$.

Bulletin technique de la S. R., 26 avril 1947. ple moteur vitesse représentée par la famille $\mathrm{T}_{1}, \mathrm{~T}_{2}, \mathrm{~T}_{3}, \ldots, \mathrm{T}_{n}$ de la figure 3 .

Les variations de rendement sont données par la courbe de rendement de la turbine.

Nous obtenons done :

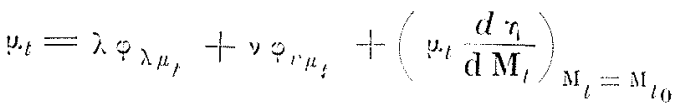

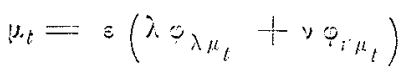

$?_{\lambda_{t} t}=$ fonction de transfert du couple moteur en fonction de louverture (la vitesse étant constante).

$i_{\mu_{t}}=$ fonction de transfert du couple moteur en fonction de la vitesse (le couple moteur étant constant).

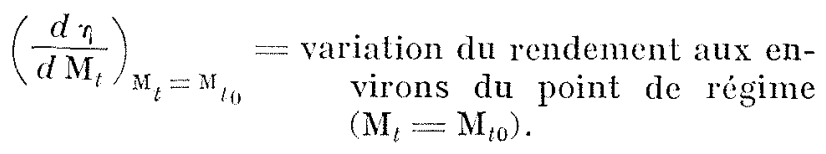

$$
\hat{c}=\frac{1}{1-\left(\frac{d r_{i}}{d M_{t}}\right)}
$$

En première approximation, nous pouvons de nouveau remplacer la caractéristique de la turbine par sa tangente aux environs du point de régine, ainsi que le présente la figure 3 : nous obtenons, en négligeant les phénomènes transitoires dans la turbine:

$$
v_{\nu, \mu_{t}}=\frac{w_{t}}{v}=\lg \alpha_{t}
$$

La fonction de transfert $\varphi_{\lambda_{\mu}}$ du couple moteur à vitesse constante est égale à la fonction du transfert de la puissance de la turbine; elle est condilionnée par la caractéristique dynamique du système hydraulique, c'est-à-dire par les phénomènes de coups de bélier. Ce qui conduit à la relation suivante (5) :

Pour une conduite a haute et moyenne chute

$$
\varphi_{\lambda \mu_{t}}=\frac{\mu_{t}}{\lambda}=1-\frac{3 \rho \lambda_{0}}{\rho \lambda_{0}+\operatorname{coth} p \frac{\mathrm{T}_{r}}{2}}
$$

(5) Voir M. CuÉnon: « Influence des phénomènes de coup de bélier sur le réglage de la vitesse des turbines hydrauliques $\gg$. La Honille Blanche, 1949, $\mathrm{n}^{\circ} 2$. 
avec :

$p=\frac{a}{2 g} \frac{v_{m}}{\mathrm{H}}=$ chiffre caractéristique de la conduite.

$\lambda_{0}=$ charge relative.

$\mathrm{T}_{\mathrm{r}}=$ temps de résonance de la conduite $\lambda=\frac{2 \mathrm{I}}{a}$

$\mathrm{I}$ = =longueur de la conduite.

$a=$ vitesse de propagation des ondes de pression.

$v_{m}=$ vitesse moyenne de l'eau dans la conduite à pleine ouverture.

Pour une conduite à faible chute

$$
\vartheta_{\lambda_{t}}=1-\frac{3 p \mathrm{~T}_{c}}{1+p \mathrm{~T}_{c}}
$$

avec :

$\mathrm{T}_{\mathrm{c}}=$ constante de temps de la conduite $=\frac{0 \lambda_{0,}}{2}$.
Pour avoir une image concrète de ce que signifie cette fonction de transfert, nous en calculons la courbe de réponse et la courbe de NYQUIST.

Pour une conduite à haute et moyenne chute:

$$
\Phi_{\lambda \mu_{t}}=1-\frac{3}{2}(1+r) r^{n}
$$

avec :

$$
\begin{gathered}
n \mathrm{~T}_{r}<t<(n+1) \mathrm{T}_{r} \quad(n=0,1,2 \ldots \infty) \\
r=\text { facteur de réflexion }=\frac{1-\rho \lambda_{0}}{1+\rho \lambda_{0}}
\end{gathered}
$$

Les figures $4 a, b, c$ représentent celte courbe de réponse pour différentes valeurs du facteur $r$.

Pour une conduite a basse chute $(\wp \rightarrow \infty)$, celte courbe de réponse dégénère en une exponentielle représentée par la figure $4 d$ :

$$
\Phi_{\lambda \mu_{t}}=1-3 e^{-t / T_{c}}
$$

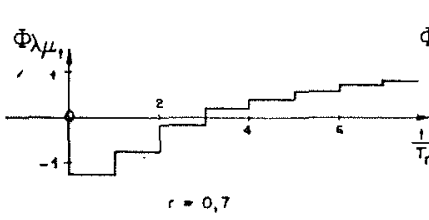

(a)

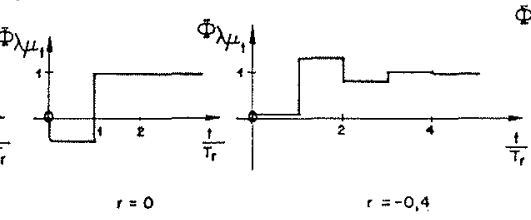

(c)

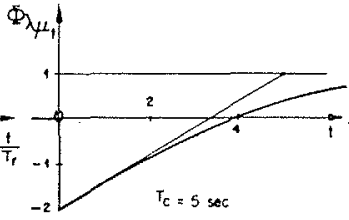

(2)

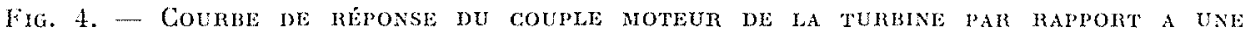
VARIATION DE SON OUVERTURE.

Pour une conduite à haute et moyenne chute, la courbe de Nyourst est un cercle, ainsi que le présente la figure $5 a$, cercle dont l'expression mathématique est la suivante :

$$
\mathrm{J}_{\lambda_{\mu_{t}}}=1-\frac{3 \rho \lambda_{0}}{\rho \lambda_{0}-j \operatorname{cotg} \zeta \frac{\mathrm{T}_{r}}{2}}
$$

Pour une turbine à basse chute, ce cercle dé- génère en un demi-cercle, ainsi que le présente la figure 50 :

$$
J_{\lambda \mu_{b}}=1-3 \frac{j \zeta \mathrm{T}_{c}}{1+j \zeta \mathrm{T}_{c}}
$$

En introduisant les fonctions de transfert des couples, nous déterminons la fonction de transfert de la vitesse en fonction des variations d'ouverture.
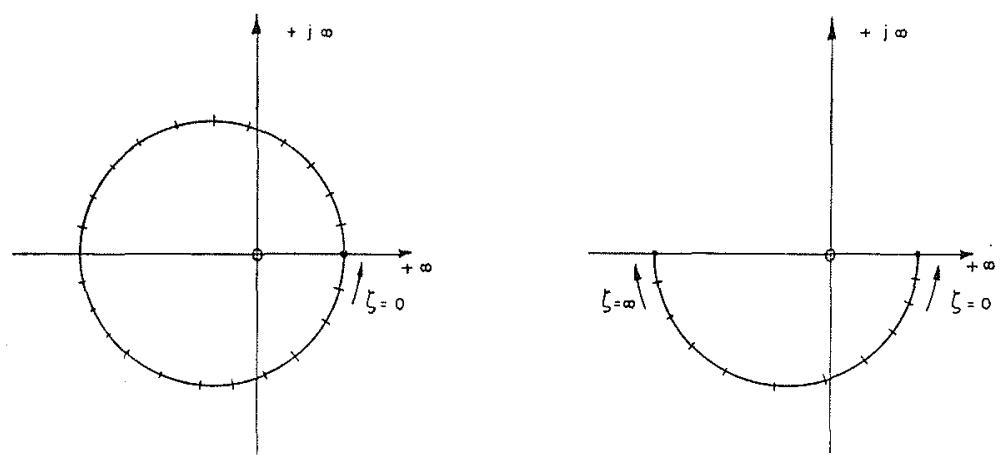

Fig. 5.

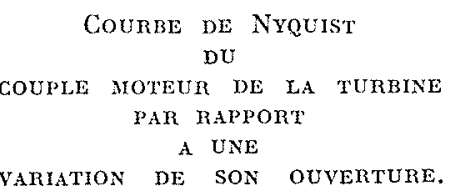




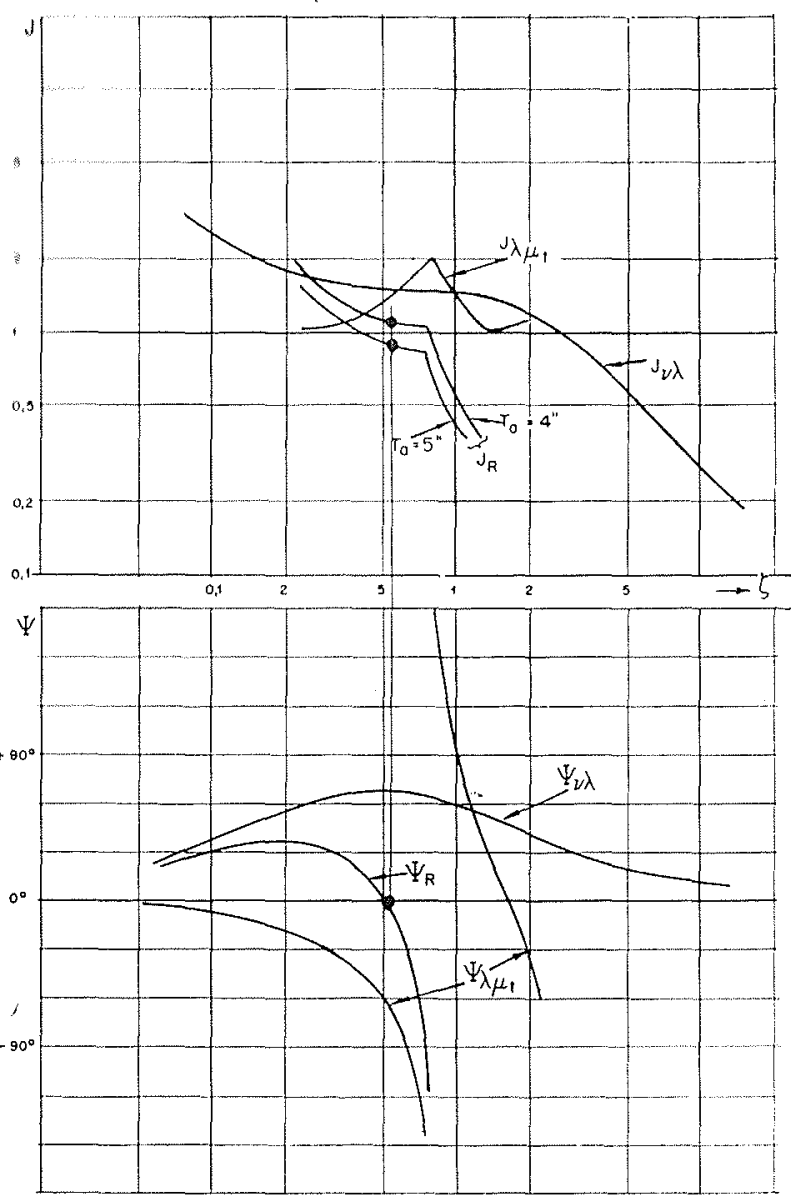

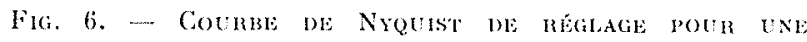
CENTRALE A HAUTE CHUTE BN MARCHE INDÉPENDANTE.

Pour un groupe en marche individuelle, nous obtenons :

$$
\varphi_{\lambda_{b}}=\frac{\varphi_{\lambda \mu_{t}} \bar{\varepsilon}}{p \mathrm{~T}_{a}+\operatorname{tg} \alpha_{e}-\varepsilon \lg \alpha_{l}}
$$

et la courbe de Nyoust des variations de la bitesse en fonction des variations de louverture:

$$
\mathrm{J}_{\lambda,}=\frac{\mathrm{J}_{\lambda \mu_{t}} \varepsilon}{j \zeta \mathbf{T}_{a}+\operatorname{tg} \alpha_{e}-\operatorname{stg} \alpha_{t}}
$$

pour un groupe en marche parallele, nous obtenons :

$$
\begin{aligned}
& \vartheta_{\lambda \nu}=\frac{\rho^{2} \vartheta_{\lambda \mu_{t}}}{\mathrm{~T}_{a}\left(\Omega^{2}+p^{2}\right)+p\left(\frac{2 \mathrm{~T}_{a}}{\mathrm{~T}_{g}}-\varepsilon \operatorname{tg} \alpha_{t}\right)} \\
& \mathrm{J}_{\lambda \nu}=\frac{j \zeta \varepsilon \mathrm{J}_{\lambda \mu_{t}}}{\mathrm{~T}_{n}\left(\Omega^{2}-\zeta^{2}\right)+j \zeta\left(\frac{2 \mathrm{~T}_{a}}{\mathrm{~T}_{l}}-\varepsilon \operatorname{tg} \alpha_{t}\right)}
\end{aligned}
$$

la courbe de NYouist de réglage est donnée par le produit des dellx courbes de Nrovist particlles. Nous obtenons donc pour un groupe, en marche indépendante :

$$
\begin{aligned}
& \mathrm{J}_{\mathrm{IR}}=\mathrm{J}_{\nu \lambda} . \mathrm{J}_{\lambda \nu}=\frac{1}{\delta} \cdot \frac{1}{j \zeta \mathrm{T}_{1}+1} \cdot \frac{1}{j \zeta \mathrm{T}_{2}} \cdot \\
& 2 \mathbf{J}_{\lambda \mu t} \\
& j \zeta \mathrm{T}_{u}+\operatorname{tg} \alpha_{e}-\varepsilon \lg \alpha_{1}
\end{aligned}
$$

et pour un groupe qui marche en parallèle avec un réseau infiniment puissant :

$$
\begin{aligned}
& J_{12}=J_{\nu \lambda} J_{\lambda b^{\prime}}=\frac{1}{\partial} \cdot \frac{1}{j \zeta \mathrm{T}_{1}+1} \cdot \frac{1}{j \zeta \mathrm{T}_{2}} . \\
& j \zeta=J_{\lambda \mu,} \\
& \mathrm{T}_{a}\left(\Omega^{2}-\zeta^{2}\right)+j \zeta\left(2 \frac{\mathrm{T}_{a t}}{\mathrm{~T}_{g}}-\varepsilon \lg \psi_{1}\right)
\end{aligned}
$$

La figure 6 représente ces courbes de Nyoust sous forme vectorielle pour des valeurs numériques correspondant à un groupe d'une centrale à haute chute en marche indépendante, en ad-
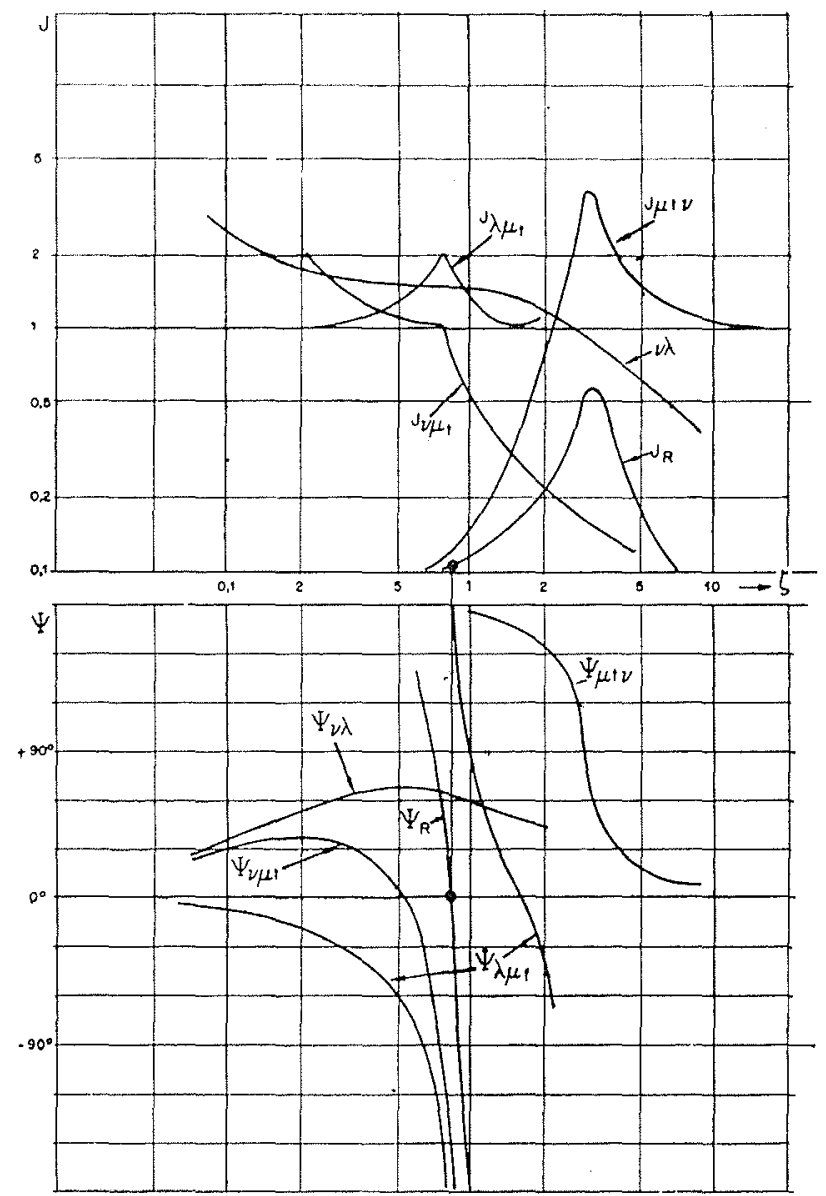

Fici. $7: Q=31 / \mathrm{s} \quad \mathrm{T}_{\mathrm{G}}=2 \mathrm{~s}$

Courbe de NYQuist de RÉglage poúr UNE CENTrale a HAUTE CHUTE MARCHANT EN PARALLĖLE. 
mettant que :

$$
\left(\operatorname{tg} \alpha_{c}-\varepsilon \operatorname{tg} \alpha_{t}\right)=0 \text { et } \quad \varepsilon=1
$$

ce qui correspond à une approximation défavorable : nous obtenons une constante d'accélération limite:

$$
\mathrm{T}_{b}=8 \mathrm{~s} .
$$

La figure 7 représente ces mèmes courbes de
Nyouist en marche en parallele; nous voyons que la stabilité est largement assurée pour une constante d'accélération de 8 secondes :

$$
(\operatorname{avec} \Omega=31 / \mathrm{s}) \quad\left(\operatorname{tg} \alpha_{t}=0 ; \quad \mathrm{T}_{\|}=2 \mathrm{~s}\right)
$$

Dans la conclusion, nous reviendrons sur la question de l'influence du PD², sur la tenue de la fréquence et de la répartition de la charge en cas de décharge particlle.

\section{III. - DISCUSSIONS DES MESURES PERMETTANT DE LIMITER LA VALEUR DU PD' AU PD' NATUREL}

\section{A. Survitesse à la suite d'une décharge brusque.}

Selon les formules que nous avons indiquées donnant le $\mathrm{PD}^{2}$ naturel $\left(\mathrm{PD}^{2}{ }_{\text {mat }}\right.$ ) et le PD² résultant du danger de survitesse $\left(\mathrm{PD}^{2}{ }^{2}\right)$, le rolor du groupe doit être surdimensionné si lingégalité suivante n'est pas satisfaite :

$$
\frac{\mathrm{PD}^{2}}{\mathrm{PD}^{2}{ }_{\mathrm{n}: \mathrm{it}}}=\frac{0,367}{\mathrm{~K}} \frac{\mathrm{T} \cos 0}{2 e+e^{2}}<1
$$

Cette condition est d'autant mieux remplie que le facteur $K$ et la survitesse admissible sont grands, et que le temps de fermeture $\mathrm{T}$ cst faible.

Le facteur $K$ est en génciral grand pour les générateurs de faible puissance ( $K$ env. 3), cl diminue avec l'augmentation de la puissance unitaire ( $K$ env. 1,5). Cependant, pour des raisons d'ordre économique, il y a intérêt à réduire le nombre des unités d'une centrale.

La réduction du $\mathrm{PD}^{2}$ par une diminution de la puissance unitaire des groupes d'une centrale n'entre pas pratiquement en ligne de compte.

Un calcul très simple montre qu'une augmentation de la survitesse admissible de $30 \%$ (valeur usuelle) à $40 \%$, toute chose restant égale, permettrait de réduire le moment d'inertic de $28 \%$. Celte mesure apparaît donc comme trìs efficace. Les générateurs sont construits de façon à pouvoir résister aux sollicitations résultant d'une vitesse d'emballement égale à 1,8 fois $l a$ vitesse normale pour les turbines Pelton, et jusqu'à 3,5 fois la vitesse nominale pour les turbines Kaplan. Aussi, il ne semble pas qu'il y ait des raisons d'ordre mécanique qui s'opposent à une élévation modérée de la valeur admissible de la survitesse à la suite d'une décharge brusque. Il paraitrait logique de prévoir differentes valeurs limites selon le genre de la construction de la turbine; cela incitera probablement les dectriciens à reviser les mesures permettant d'éviter le danger des surtensions occasionnées par ces survitesses. Il est en effet possible d'y remédier par une action judicieuse du système d'excitation du générateur en cas de décharge brusque.

Pour les turbines Francrs qui ne sont pas munies de déchargeurs, et les turbines Kaplan, le temps de fermeture $\mathrm{T}$ dépend des caractéristiques du systeme hydraulique. Une diminution du temps caractéristique, et partant du temps de fermeture 'T', peut être obtenue soit par un raceourcissoment de la conduite alimentant la turbine, soil par une diminution de la vitesse moyenne d'écoulement de l'eau en agrandissant le diamètre de la conduite. Pour les centrales à basse chute, la longueur de la conduite est imposée par les dimensions de l'installation ou par les conditions géographiques du canal d'amenée de l'ean. Il n'est pas possible de les modifier à volonté. Un agrandissement du diamètre de la conduite conduirail à un coût supérieur à celui d'une augmenlation du $\mathrm{PD}^{2}$ que cet agrandissenent permettrait deconomiser. Pour les centralcs à haute chute, la conduite forcée pourrait être raccourcie artificiellement en construisant des chambres d'équilibre intermédiaires. La surpression admissible peut être élevée par un renforcement des parois de la conduite. La vitesse d'écoulement de l'eau peut être diminuée par une augmentation du diamètre de la conduite. Il semble cependant que dans ce cas également, l'économie du $\mathrm{PD}^{2}$ ne compenserait pas l'augmentation du coût de la conduite. En conclusion, il ne paraît pas que, d'une façon générale, la diminution du temps de fermeture soit un moyen efficace pour diminuer le moment d'inertie. 
La situation se présente différemment pour les installations à chute moyenne équipées de turbines Fraveis avec déchargeur ou orifice compensateur. Lors d'une fermeture de la lurbine, l'eau peut s'échapper en contournant la turbine, de sorte que les aubes de l'appareil dirceteur peuvent être actionnées rapidement sans qu'il y ait à redouter des surpressions.

Les turbines PELTON pour les installations à haute chute sont actuellement toujours équipées d'un déflecteur qui peut intervenir rapidement en cas de décharge brusque sans aucun inconvénient pour la conduite forcée. Par contre, la vitesse de réaction du pointeau dont il faut tenir compte pour le réglage de la vitesse est limitée par l'inertie de l'eau dans la conduite.

Il apparait done que pour les installations à haute chute il n'est pratiquement jamais nécessaire d'augmenter artificiellement lo $\mathrm{PD}^{2}$ pour obvier au danger de survitesse au cas de décharge brusque. Pour les installations à chute moyenne, une étude comparative de prix permettra de déterminer quelle est la solution la plus avantageuse : augmentation du $\mathrm{PD}^{2}$ ou construction d'un orifice compensateur ou d'un déchargeur.

Comme mesure pour limiter la survilesse, il a été proposé d'enclencher aux bornes du générateur une résistance hydraulique en cas de décharge brusque (6). La valeur de cette résistance serait ensuite augmentée progressivement en sorte que le générateur soit déchargé peu à peu de facon que, mème avec un temps de fermeture peu élevé et sans augmentation artificielle du $\mathrm{PD}^{2}$ tout danger de surpression et de survitesse soit éliminé.

Cependant, il ne semble pas que l'équipement des générateurs avec des résistances hydrauliques revienne beaucoup meilleur marché que l'adjonction d'un volant. Cette dernière mesure a en outre l'avantage d'offrir une plus grande sécurité de marche.

Nous tirons des considérations précédentes les conclusions suivantes :

Une augmentation du PD", eu égard au danger de survitesse en cas de décharge brusque, ne paraît pas entrer en ligne de compte pour les groupes entraunés par une turbine PELton, ou une turbine Francis avec déchargeur automatique ou orifice compensateur. Il semble de facon générale qu'il est plus avantageux au point de vue économique d'éviter également une telle augmentation pour les turbines Francrs sans déchargeur et pour les turbines Kaplan ou à hélice, en tolérant une marge plus grande de sur-

(6) F. CaHeN : « Le freinage des groupes hydro-électriques $\gg$.

Revue générale d'Electricité, juin 1947. vitesse. Cette mesure parait d'autant plus raisonnable que les groupes de ce genre sont construits de façon à pouvoir supporter des survitesses plus élevées que celles qui ont été admises généralement jusqu'à aujourd'hui.

Ces quelques remarques ne doivent être considérées que comme une orientation générale. Elles ne peuvent ni ne doivent remplacer une étude comparée des différentes mesures auxquelles nous avait fait allusion pour chaque cas particulier en les considérant surtout sous leur angle économique, lors du projet de nouvelles installations.

\section{B. Stabilité du réglage de vitesse.}

L'application du critère de Nyourst montre clairement l’influence très défavorable des phénomènes de coup de bélier sur la stabilité du réglage de vitesse, et cela d'autant plus que la charge du groupe, le temps de résonance $\mathrm{T}_{r}$ ou la constante de temps $\mathrm{T}_{6}$ de la conduite sont élevés. Il ressort que l'on ne peut espérer diminuer d'une facon appréciable le $\mathrm{PD}^{2}$ nécessaire à la stabilité du réglage de vitesse par un perfectionnement du dispositif de réglage, par exemple par une diminution du temps de réaction $\mathrm{T}_{1}$ ou par un régleur ou accéléromètre à fréquence propre très élevée, car cela n'a pratiquement aucune influence sur la cause même de l'instabilite, qui est l'inertie de l'eau dans la conduite.

Nous devons distinguer les conditions de marche suivantes :

1. - Marche EN PARALLìle AVEC UN RÉSEAU INFINIMENT PUISSANT

a) Générateur accouplé directement au résean :

Il peut se produire un phénomène de résonance si la fréquence propre du générateur coïncide avec celle des ondes de pression de la conduite, ce qui peut expliquer le fait que certains générateurs stables en marche individuelle deviennent instables en marche parallèle. Mais ce cas est relativement rare, et d'une façon générale, on peut conclure que le $\mathrm{PD}^{2}$ naturel est toujours suffisant pour assurer dans ce cas la stabilité du réglage de vitesse.

\section{b) Le générateur est accouplé par l'intermé- diaire d'une longue ligne :}

Le problème de la stabilité électrique qui se pose alors doit être soigneusement distingué du problème de la stabilité du réglage de vitesse; différentes mesures, telles que l'excitation série, le réglage de l'excitation ou du couple moteur 
en fonction du déphasage interne, permettent d'assurer artificiellement la stabilité électrique; la valeur du PD' n'intervient que pour la détermination de la fréquence propre des oscillations mais n'a pratiquement qu'une faible influence sur la stabilité proprement dite. On peut remarquer cependant qu'un faible PD'2 rend plus difficile un réenclenchement rapide; en effet, une rupture du synchronisme, le glissement prend alors une valeur qui risque de devenir trop élevée.

\section{c) Le générateur est accouplé au moyen d'un"} ligne ayant une forte résistance ohmique:

Ce cas est particulièrement defavorable pour la stabilité électrique; pour une certaine valeur critique de la résistance, le générateur se met: osciller sans qu'il soit possible de le stabiliser artificiellement. Une augmentation artificielle du moment d'inertie n'améliore pratiquement pas la stabilité dans ce cas particulier.

\section{2. -.. MARGhe individuella}

\section{a) Charge avec des moteurs:}

Pour la détermination de la stabilité, on peut considérer que le PD2 des masses tourmantes s'additionne au PD² du générateur et que, d'autre part, les moleurs ont souvent une charge qui augmente avec leur vitesse, par exemple ceux qui entraînent les compresseurs ou les ventilateurs. Il résulte que, dans ce cas, le PD² naturel est suffisant pour assurer la stabilité du reiglage à condition que l'accouplement entre moteur et générateur soit suffisamment rigide.

\section{b) Charge a caractère ohmique el inductif.} Sans réglage de tension :

La tension augmente avec la fréquence, en particulier si les excitatrices principales et auxiliaires sont en bout d'arbre; il en résulte que la charge augmente également, la stabilité est assurée sans qu'il soit nécessaire d'augmenter dans une grande marge le $\mathrm{PD}^{2}$ par rapport au $\mathrm{PD}$ ? naturel; cependant, les variations de tension peuvent être trop élevées pour être admissibles pour la pratique.

\section{Réglage à tension constante :}

En admettant que le réglage de la tension s'effectue instantanément, la puissance active élcctrique consommée est maintenue constante ou même peut diminuer lorsque la fréquence augmente, le couple décroît avec la vitesse; ce cas est particulièrement défavorable pour la stabilité du réglage de vitesse. Le PD nécessaire pour obtenir un réglage slable dans ce cas peut atteindre un multiple du PD" naturel. L'augmentation du PD' telle qu'elle peut entrer en ligne de compte pratiquement s'avère insuffisante pour garantir un réglage stable.

\section{Réglage fréquence-tension (7) :}

En insérant la résistance de réglage d'un rógulateur de fréquence statique dans le circuit de mesure du régulateur de tension, la tension est rendue linéairement dépendante de la fréquence, c'est-ádire qu'il est possible de fixer à volonté la pente de la caractéristique du générateur et d'obtenir un réglage stable avec un $\mathrm{PD}^{2}$ relativement trop faible, ainsi que cela a été confirmé par l'expérience dans les centrales de Ryburg-Schworstadt et de Reckingen en Suisse.

Cette stabilisation est obtenue aux dépens du maintien rigoureux de la tension. Si l'on veut éviter les écarts permanents de tension qui seraient la conséquence d'écarts permanents de fréquence, et si l'on craint en particulier, en cas de marche en parallèle, que ce dispositif ne conduise à des variations de charge réactive, il suffit de rendre le statisme du régulateur de fréquence passager et faire en sorte que son action s'annule en régime permanent, ce qui peut être obtenu par différents artifices.

\section{3. - Marche en parartide avec Un réseau DE PUISSANCE LIMITÉE}

Co cas comprend tous les cas intermédiaires entre les cas 1 et 2 et nécessite une étude particulière dans les différents cas concrets selon la structure du réseau en question. Cependant, on peut remarquer que plus l'interconnexion se développe, plus les points d'interconnexion sont nombreux, plus on se rapproche du cas 1 . I. cas 2 est toujours plus défavorable que le cas 3 . Il est légitime de se limiter en première approximation à l'étude de la stabilité en marche individuelle, tout en faisant la réserve que cela représente dans certains cas des conditions trop défavorables pour servir de norme.

(7) R. Kexter : «La stabilisation du réglage de la vitesse de groupes générateurs à charge indépendante de la fréquence 》.

Revue Brown Boveri, juin-juillet 1947, pages 99-104. 


\section{IV. - DISCUSSION DE QUELQUES CAS CONCRETS}

L'augmentation du PD² qu'il est nécessaire de prévoir est parfois du même ordre de grandeur que celui dı PD: naturel. Voici, à titre d'exemple, ee qu'il en est pour quelques ecntrales de petite et moyenne puissance et à moyenne el grande vilesse :

\begin{tabular}{|c|c|c|c|c|}
\hline Centrales & A & B & C & $\mathrm{D}$ \\
\hline $\mathrm{PD}^{2}$ du rotor $\ldots \ldots \ldots \operatorname{tm}^{2}$ & 22 & 30 & 1,2 & 12 \\
\hline $\mathrm{PD}^{2}$ du volant........tm² & 43 & 36 & 1,3 & 10 \\
\hline
\end{tabular}

Si l'on veut supprimer le danger de survitesse par l'enclenchement de résistances hydrauliques, et si l'on tient compte de l'ensemble du disposi-
Lif que cela nécessite (interupteur, conduite électrique, résistance hydraulique, relais, etc.), il n'en résulte pas une économie appréciable par rapport à une limitation de la survitesse à l'aide d'une augmentation du PD'. Ce second moyen offe lavantage de garantir une plus grande sécurité de marche.

A titre de référence, nous avons déterminé les conditions de stabilité dans trois cas, pour une centrale à haute chute, une centrale à chute moyenne et une centrale à basse chute.

Les calculs ont été faits en admettant une puissance consommée constante, ce qui suppose une charge indépendante de la fréquence et un réglage a tension constante infiniment rapide. Cela représente des conditions de réglage particulièrement défavorables. Les résultats auxquels ces calculs conduisent sont indiqués dans le tableau ci dessous :

TABLEAU DES DONNÉES TECHNIQUES DES TROIS CENTRALES CHOISIES COMME RERERENCE

\begin{tabular}{|c|c|c|c|}
\hline Type de centrale & $\begin{array}{l}\text { Halule chule } \\
\mathrm{A}\end{array}$ & $\begin{array}{c}\text { Chute moyenne } \\
\mathrm{B}\end{array}$ & Basse chute \\
\hline Débit $Q(a ̀$ pleine charge $) \ldots \ldots \ldots \ldots\left(\mathrm{m}^{3} / \mathrm{s}\right)$ & 3 & 17 & 255 \\
\hline Longueur de la conduile $\mathrm{L} \ldots \ldots \ldots \ldots \ldots(\mathrm{m})$ & 1930 & 858 & $\begin{array}{l}70 \\
\text { avee laspirateur }\end{array}$ \\
\hline Hauteur de chute $H \ldots \ldots \ldots \ldots$ (n) & 996 & 185 & $7-10$ \\
\hline Section moyenne $S \ldots \ldots \ldots \ldots \ldots \ldots\left(\mathrm{m}^{\circ}\right)$ & 0,66 & 4,7 & 32 \\
\hline Vitesse moyenne $v_{n} \ldots \ldots \ldots \ldots \ldots(\mathrm{m} / \mathrm{s})$ & 4,5 & 3,7 & 8 \\
\hline Période propre de la conduite $\mathrm{T}, \ldots \ldots \ldots$ (s) & 3,8 & 1,7 & - \\
\hline Constante de temps de la conduite $\mathrm{T}_{\ldots} . \ldots$ (s) & - & - & 2,8 \\
\hline Grandeur caractéristique de la conduile... & 0,23 & 1,03 & $\ldots$ \\
\hline 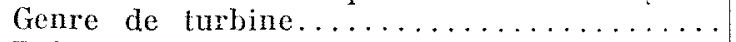 & PEL'TON & Francts & KAPLAN \\
\hline Puissance de la turbine.............. (PS) & 36000 & 7500 & 24800 \\
\hline Vitesse $\ldots \ldots \ldots \ldots \ldots \ldots \ldots \ldots \ldots \ldots \ldots \ldots \ldots \ldots$ & 500 & 500 & 75 \\
\hline $\left.\mathrm{PD}^{2} \ldots \ldots \ldots \ldots \ldots \ldots \ldots \ldots \ldots \ldots \ldots \ldots \mathrm{tm}^{2}\right)$ & 202 & 110 & 8800 \\
\hline Puissance du générateur N....... (kVA) & 30000 & 6500 & 22000 \\
\hline $\mathrm{K}=\frac{\mathrm{PD}^{2} n^{2}}{\mathrm{~N} 10^{3}}, \ldots \ldots \ldots \ldots \ldots \ldots \ldots \ldots \ldots$ & 1,7 & 1,65 & 2,26 \\
\hline Constante d'accélération $\mathrm{T}_{4} \ldots \ldots \ldots \ldots$ (s) & 5,2 & 5,8 & 7,5 \\
\hline Réglage instable $\mathrm{T}_{a} \ldots \ldots \ldots \ldots \ldots \ldots \ldots$ (s) & 5 & 4 & 8 \\
\hline Réglage stable $\mathrm{T}_{a} \ldots \ldots \ldots \ldots \ldots \ldots$ (s) & 8 & 5 & 13 \\
\hline
\end{tabular}

\section{LÉGENDE}

$v_{m}=$ vitesse moyenne de l'eau dans la conduite forcée :

$$
v_{m}=\frac{\mathrm{Q}}{\mathrm{L}} \Sigma \frac{\Delta \mathrm{L}_{i}}{\mathrm{~S}_{i}}(\mathrm{~m} / \mathrm{s}) \text {. }
$$

$\mathrm{Q}=\operatorname{débit}\left(\mathrm{m}^{3} / \mathrm{s}\right)$.

$\mathrm{L}=$ longueur totale de la conduite $(\mathrm{m})$.
$\Delta \mathrm{L}_{i}=$ longueur des différents tronçons (m).

$\mathrm{S}_{i}=$ section des différents tronçons $\left(\mathrm{m}^{2}\right)$.

$a_{n}=$ vitesse moyenne de propagation des ondes de pression $(\mathrm{m} / \mathrm{s})$ :

$$
a_{m}=\frac{\mathrm{L}}{\Sigma \frac{\Delta \mathrm{L}_{i}}{a_{i}}}
$$


$a_{i}=$ vitesse de propagation dans les différents tronçons $(\mathrm{m} / \mathrm{s})$.

$p=$ grandeur caractéristique de la conduite :

$$
\rho=\frac{a_{m} v_{m}}{2 g \mathrm{H}}
$$

$\mathrm{H}=$ hauteur de chute (m).
${ }^{\top} \mathrm{T}_{r}=$ période propre de la conduite $=\frac{2 \mathrm{~L}}{a_{m}}(\mathrm{~s})$. $\mathrm{T}_{c}=$ constante de temps de la conduite

$$
=\frac{\mathrm{L} v_{m}}{2 g \mathrm{H}}(\mathrm{s})
$$

Il ressort de cette étude comparative qu'avec
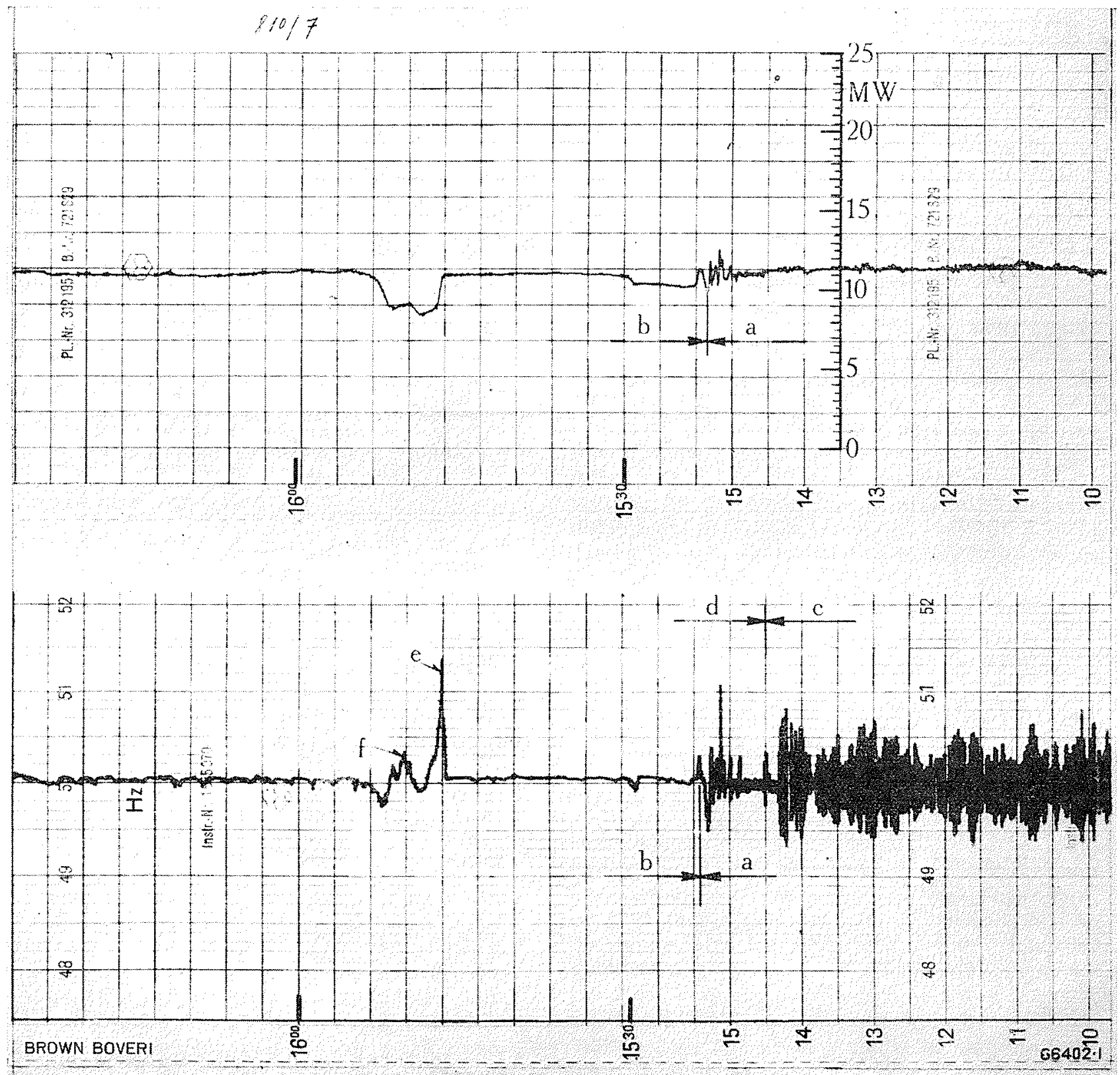

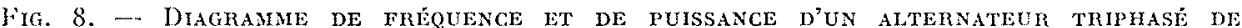
$22000 \mathrm{KVA}, 11000 \mathrm{~V}, 75 \mathrm{TmS} / \mathrm{MN}, 50 \mathrm{~Hz}$, ALIMENTANT UN FOUM ELECTRYUE, AVIEC ET SANS STABHISATEUR ÉLECTRIOUE :

a) avance lente de la bande enregistreuse, c) sans stabilisation électrique,

$b$ ) avance rapide de la bande enregis- $d$ ) avec stabilisation électrique, treuse.

e) et f) variations brusques de charge. 
des PD-2 tels qu'ils ont été choisis, la marche du groupe dans ces conditions particulières est stable pour la centrale B (à moyenne chute), à la limite de la stabilité pour la centrale $A$ (à haute chute), instable pour la centrale $B$ (à basse chute). Dans ce dernier cas, ce résultat est confirmé par des essais (8) dont la figure 8 donne le résultat; lorsque le groupe est en marche indépendante et alimente une usine chimique, le diagramme des vitesses indique des oscillations de réglage très caractéristiques. Cependant, un réglage de vitesse parfaitement stable a pu être obtenu par une action en fonction de la fréquence sur le réglage de tension, ainsi que le montre la figure 8.

L'étude de ces quelques cas particuliers prouve que le $P D^{2}$ choisi est à la limite de celui qui est nécessaire pour assurer un réglage de vitesse stable dans le cas défavorable d'une marche à tension constante sur une charge indépendante de la fréquence. Mais, d'autre part, clle a confirmé ce que des considérations théoriques avaient laissé prévoir, à savoir qu'une stabilisation obtenue par un réglage adéquat de la tension peut ètre plus économique que celle obtenue par une augmentation du $\mathrm{PD}^{2}$, sans compromettre la sécurité de l'installation : si le réglage de la tension fait défaut, les oscillations du réglage de vitesse ne mettent pas en danger l'installation tout en étant intolérables pour un régime permanent.

\section{V. - Conclusion}

Les constructeurs de générateurs se trouvent souvent placés devant l'exigence de prévoir pour leur machine un moment d'inertie supérieur à celui auquel les conduit leur propre critère, soit pour limiter le danger de survitesse à la suite d'une brusque décharge, soit pour assurer un riglage de vitesse stable. Dans ces deux cas, ce qui est déterminant, c'est la vitesse d'action du servomoteur, celle-ci étant limitée elle-même par la nécessité de tenir compte de l'inertie de l'eau dans la conduite alimentant la turbine.

Si l'on considère les deux possibilités suivantes : réduire le temps de fermelure de facon à rendre superflue une augmentation du $\mathrm{PD}^{2}$ en changeant la disposition du systeme hydraulique, ou bien, accepter le temps de fermeture imposé et élever le moment d'inertie de facon à satisfaire aux conditions de survitesse el de sta-

(1) Voir R. KetLen : « La stabilisation du réglage de la vitesse de groupes générateurs à charge indépendante de la fréquence $\gg$.

Revue Brown Boveri, juin-juillet 1947, page 103. bilité, il semble que cette deuxième alternative apparait en règle générale comme étant la plus íconomique.

Cependant, une étude plus approfondie des conditions de survitesse montre qu'elles n'imposent aucune augmentation du moment dinertie pour les turbines Pelton et que, pour les turbines Francis, munies de déchargeur automatique ou d'orifice compensateur, une étude comparative des coûts des dispositifs de stabilisation est nécessaire pour déterminer la solution la plus économique.

Dans les autres cas, en parliculier pour les turbines Kaplan, cette augmentation peut être réduite, sinon supprimée, en admettant à la suite d'une décharge brusque une survitesse légèrement supérieure à celle qui est prévue généralement. Si l'on tient compte de la vitesse d'emballement relativement élevée pour laquelle ces machines sont calculées, une augmentation modérée de la survitesse admissible à la suite d'une décharge brusque et totale apparait comme raisonnable.

Si l'on fait exception de certains cas d'instabilité lors d'une marche en parallele avec un réseau puissant en faveur desquels une augmentation du $\mathrm{PD}^{2}$ ne serait que d'un faible secours, le cas le plus critique pour la stabilité du réglage de vitesse est celui d'un générateur chargé sur une résistance ohmique, avec réglage à tension constante. Cependant, comme la théorie et la pratique l'ont montré, il est possible de remédier at l'instabilité qui peut se produire dans ce cas, sans augmenter artificiellement le moment d'inertie, par un réglage judicieux de la tension en fonction de la fréquence.

Il n'est peut-être pas superflu de préciser qu'il ne faut pas espérer une diminution de la survitesse ou une amélioration de la stabilite par un raffinement ou une plus grande rapidité d'action du dispositif de réglage de la turbine. Le temps de fermeture est en effet imposé par les caractéristiques du système hydraulique.

Nous avons négligé d'examiner jusqu'à présent, dans notre exposé, l'influence du PD' sur le comportement du groupe lors de variation de charge partielle. En marche en parallèle, la répartition de la charge entre les différents groupes interconnectés a la suite de brusques variations de la consommation s'opère passagèrement, avant que le dispositif de réglage des turbines ait le temps d'intervenir, en raison des constantes d'accélération des groupes. Si ces constantes sont trop divergentes et que l'un des groupes se trouve à la limite de sa stabilité électrique, il pent se produire un décrochement intempestif à la suite d'une variation brusque de charge. A ce point de vue, les moments d'inertie devraient ètre choi- 
sis de facon à uniformiser ces constantes d'accilération. Cependant, dans certains cas, lors de l'interconnexion de réseaux de même puissance, un moyen très efficace pour parer aux troubles causés par un à-coup de charge est donné par le réglage fréquence-puissance direct, qui contròle automatiquement la répartition de la charge entre réseaux interconnectés et en assure la stabilité. Les expériences faites à la centrale de la Dixence confirment cette conclusion que la thcorie laissait prévoir.

En marche sur réseau séparé, ainsi que l'a fait remarquer J. Chenais (9), la valeur $P^{2} D^{2}$ a une influence directe sur la tenue de fréquence lors des variations de charge partielle. Plus le PD:- est faible, plus les variations de fréquence à la suite d'un à-coup de charge seront importantes. Cependant, cet inconvénient est en partic compensé par le fait que les écarts de fréquence sont alors de plus courtes durées. Wautre part, avec le développement de l'interconnexion, la marche sur réseau séparé apparait de plus en plus comme exceptionnelle. Elle peut cependant se produire en particulier pour l'alimentation d'usines chimiques, ou de fours électriques, qui n'exigent pas une tenue rigoureuse de la fréqence ou du fait de l'éloignement entre les centres de production électrique. Lorsque le réseau alimente un réseau industriel comportant des moteurs plus sensibles aux variations de fréquence, leur moment d'inertic s'ajoute à celui du générateur et limite Iui aussi les variations de fréquence. Enfin, de mème que la stabilité,

(9) J. Chenas : « Détermination du PDz de groupe hydro-ćlectrique $\gg$.

Bullelin de la Société Francaise des Electriciens, nov. 1947 . la tenue de la fréquence peut être améliorée, en cas de nécessité, par un réglage judicieux de la tension en fonction de la fréquence.

Ainsi, il apparait que, pour parer aux inconvénients résultant d'une variation de charge partielle soit en marche en parallele, soit en marche individuelle, il m'y a pas liea, d'une facon générale, d'élever la valeur du moment d'inertie du groupe.

En résumé, ainsi que le déclare la Revue Brown Boveri (10) : «On peut conclure que, dans de nombreux cas, il serait opportun, au point de vue économique, d'admettre une valeur plus élevée de survitesse lors de suppression de la charge totale pour pouvoir si possible réduire le moment d'incrtie. De mème, il est possible, sans faire appel à une augmentation du moment d'inertie, de résoudre le problème de la stabilité du réglage de vitesse dans le cas crilique d'une charge indépendante de la fréquence en prenant des dispositions pour agir passagèrement sur le réglage de la tension de manière à faire varier la charge avec la fréquence, solution qui est en général beaucoup plus économique. $\gg$

Ainsi, l'électricien oflre des moyens de réglage permettant souvent de réduire le $\mathrm{PD}^{2}$ considéré comme nécessaire par le turbinier. Il est done souhaitable que turbiniers et électriciens étudient ensemble le problème de la détermination du PD² pour être ensuite en mesure de mettre à disposition de l'exploitant la solution la plus satisfaisante tant au point de vue économique que technique.

(10) E. GUNThandT: «Sur les alternateurs de grande puissance pour centrale hydraulique ».

Rerue Brown Boveri, juillet-août 1949 .

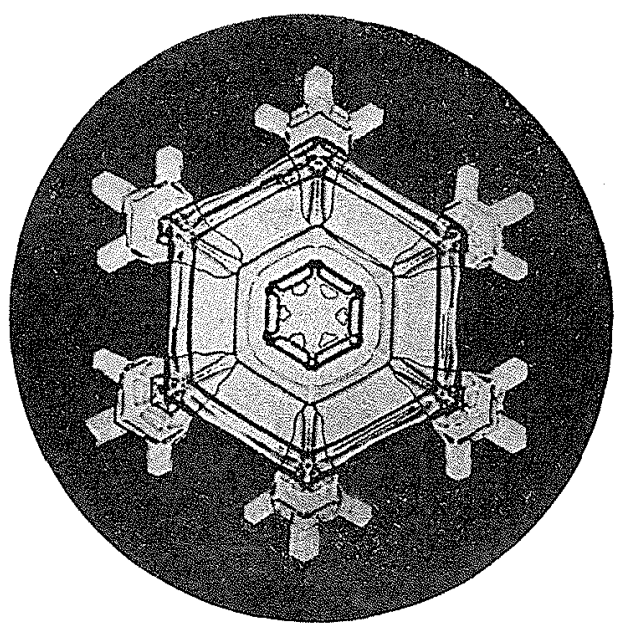

